\title{
Response vs. Perception ${ }^{\text {i }}$
}

\section{An Enduring Question Revisited in the Domain of Context Effects}

\author{
Willam Schneiderman ${ }^{2}$
}

The University of Alberta

\section{Melvin Manis}

The University of Michigan and Ann Arbor Veterans Administration Hospital

Three experiments were conducted in which college students read, and then attempted to match, a series of written descriptive passages with the referent photographs on which they were based; the photographs showed the face of an actor, representing a variety of emotional expressions. In Experiment I, subjects provided with a series of context passages depicting a narrow range of emotions (neither pleasant nor unpleasant) chose "matches" having more extreme pleasantness values than did subjects provided with context passages depicting a wide range of descriptions on the pleasantness dimension when responding to test descriptions embedded within the context series. In Experiments II and III, contrast effects were obtained; subjects who had read mostly unpleasant context passages chose more pleasant referents in response to neutral test descriptions than did those who had read mostly pleasant descriptions. The results of all three experiments suggested that these effects were mediated in large part by a response bias, the tendency to use each response alternative with roughly equal frequency. In Experiments II and III, there was suggestive evidence for the possibility that a more central (or perceptual) mechanism may also have contributed to the observed results.

The context in which a stimulus is embedded has repeatedly been shown to affect the judgmental response evoked by that stimulus (Helson, 1964); judgments are normally displaced away from the contextual stimulation-a

\footnotetext{
'All statements herein are those of the authors and do not necessarily represent the opinions or policy of the Veterans Administration.

'Requests for reprints should be addressed to Dr. William Schneiderman, Department of Psy* chology, The University of Alberta, Edmonton, Alberta, Canada T6G 2 E9.
} 
contrast effect. For example, Campbell, Hunt, and Lewis (1958) found that judges exposed to predominantly "high pathology" definitions of vocabulary words rated midscale definitions as less pathological than judges exposed to predominantly "low pathology" definitions. Examples of this phenomenon have been demonstrated with judgments of morality (Parducci, 1968), line length (Krantz and Campbell, 1961), and facial expression (Manis, 1971).

The purpose of this research was to gain some understanding of the process(es) underlying the contrast effect, for the social judgment literature includes a long-standing and still active controversy concerning the origins of this phenomenon (Simpson and Ostrom, 1976; Erdelyi, 1974; Krupat, 1974). Some investigators have suggested that the contrast effect can easily be explained in terms of peripheral mechanisms, while others invoke more central determinants. Peripheral explanations often focus on "linguistic" processes, for they emphasize the possibility that context effects may operate by changing the manner in which the individual reports on his subjective experience. Other theorists have suggested that contextual factors operate at a more central level, and may, for example, affect the respondent's subjective or "perceptual" experience.

The linguistic (or peripheral) hypothesis implies that context effects may be systematically influenced by the response mode that is used to index subjective experience. For example, the subjects in Manis' (1967) study who simply rated the pleasantness of various descriptions of facial expression may have been subject to the response level (linguistic) processes that are referred to above. When these subjects rated mostly pleasant descriptions, they may have redefined the response categories of "very pleasant" and "very unpleasant" to accord with the endpoints of the presented range of descriptions. A neutral test description may therefore have been rated as unpleasant (an apparent contrast effect), even if the respondent's subjective experience of pleasantness had not been altered. This kind of repositioning of the response scale is particularly likely when the response language is either novel or relativistic. When the response language is more firmly anchored, the possibility of this kind of linguistic effect is substantially reduced.

In an effort to eliminate the ambiguity that is almost inevitably involved in traditional rating scale methodologies, Manis (1967) employed a decoding paradigm involving response alternatives that seemed likely to be more firmly anchored than rating scale categories. In this procedure, the subject reads a description, and instead of rating its apparent "pleasantness," is presented with a large set of photographs from which he attempts to select the particular photograph that the passage was based upon. The experimenter then infers the "perceived pleasantness" of the description by noting the location of the selected photograph (pleasant-unpleasant), using 
a set of previously established norms. In Manis' (1967) study of context effects, he found the usual contrast phenomenon, whether the subjects responded using traditional rating scale procedures, or the decoding technique that is described above; in both cases, the respondents displaced the test stimuli away from the contextual stimuli. Since this displacement effect appeared to be unaffected by the response modality that was employed, Manis concluded that the contrast effect was probably a reflection of some central process, which he labeled as "perceptual," in accordance with the conventions of this area (Upshaw, 1967). ${ }^{3}$

While the decoding paradigm seemed to guard against the possibility of one response bias, category repositioning, it did not control for another plausible bias - the tendency to use each of the available response alternatives with roughly equal frequency (Parducci, 1974). This kind of response bias is evident when a student who guesses on a multiple choice item balances the number of $\mathrm{A}, \mathrm{B}, \mathrm{C}$, and $\mathrm{D}$ responses by choosing the underused alternative. In the Manis (1967) study, among respondents who had mainly decoded pleasant descriptions, the photographs of unpleasant emotions would probably have been selected as the correct response (the "'match") quite infrequently. If these subjects tended to use each of the response alternatives with roughly equal frequency, neutral test descriptions would acquire an increased likelihood of eliciting choices from these unpleasant (and previously underchosen) photographs. If this equal frequency tendency were in fact operative, certain response alternatives would acquire an enhanced probability of being selected as "matches" for the test descriptions, due to the infrequency with which they were chosen in the past. This equal frequency response bias could have generated the Manis (1967) results.

The present research attempted to explore the locus (central vs. peripheral) of various context effects. The methodology that was devised sought to control for the two response factors that are discussed above (category repositioning and the equal frequency bias), through variations in the decoding procedure.

${ }^{3}$ Strictly speaking, it is impossible to demonstrate that a given effect is unequivocally "perceptual," since we cannot directly examine the respondent's perceptual field. To overcome this dilemma many investigators have sought to demonstrate that a given effect (e.g., contrast) can be obtained using a variety of independent response measures. If this can be done, it is taken as a sign that the effect in question probably reflects some relatively central process that is "upstream" from the particular responses actually observed. And by convention, central processes or this sort are often termed "perceptual"; this convention, together with the reasoning that underlies it, represents the main justification for invoking the term "perceptual" in the present paper. 


\section{EXPERIMENT I}

This study was designed to explore stimulus variance as a context variable. Respondents were presented with (a) descriptions of emotional poses that were neutral with respect to pleasantness, (b) descriptions that reflected the full range of emotional expressions, or (c) descriptions based exclusively on faces that were extreme with respect to the pleasantness dimension. In this way, the variance of the stimuli in the experiment (i.e., the descriptions) was manipulated, while the mean value of the stimulus array remained constant.

Variance manipulations may produce effects that parallel the results normally generated through variations in central tendency. That is, in both cases, there may be a judgment displacement in a direction that is opposite to the bias provided by the context stimuli. For example, subjects who have received a narrow variance of stimulation may produce more extreme responses to test stimuli than do subjects who have been exposed to a wide variance of stimuli, and vice versa. Experiment I was designed to assess this conjecture, and to establish the locus (central vs. peripheral) of the effects that were obtained.

\section{Method}

\section{Subjects}

Forty-eight students in introductory psychology courses at the University of Michigan participated in this study. Subjects were run in groups of one to twelve.

\section{Apparatus}

Photographs. The Frois-Wittman (1930) photographs were used as response alternatives (referents). Forty-eight of these were selected and presented on two pages, each containing twenty-four photographs; these pages were labeled A and B. Each page was constructed as follows:

1. There were six rows and four columns of photographs.

2. Under each photograph was placed the identification number from the Frois-Wittman series.

3. Each page contained photographs spanning the pleasantness dimension as scaled by Schlosberg (1952) and spaced at roughly equal intervals along this dimension.

4. The row and column positions of each photograph on each page were determined randomly. Thus, in most instances, photographs with 
similar pleasantness values would be in different positions on Pages $\mathrm{A}$ and B.

Descriptions. Descriptions of the photographs were obtained from a previous study (Manis \& Armstrong, 1971). Subjects in that study had been instructed to "Write your impressions of the mood, feelings, or thoughts of the actor in each picture, or give an indication of how you would feel if you were making that particular expression," and to "... do this in such a way as to convey enough information so that someone else would know which picture you wrote about." Typical descriptions were: "Intense concentration. Seriousness. Desire to stamp indelibly upon his mind what he is seeing. Like a medical student seeing his first operation," "The man is guffawing," and "Like a high school teacher waiting for his class to shut up so he can get back to his teaching." Descriptions were then assembled into booklets.

\section{Procedure}

Booklets and photograph pages were placed before the subjects, and they were instructed as follows: "In this booklet are descriptions of the accompanying faces. Your job is to try to decide which face the description refers to. Please write the number of the face you think the description refers to in the blank space following the description. Next to the blank space will be the letter A or the letter B. This tells you which page of faces the correct answer can be found on. If it is an A, look on the page marked group $\mathrm{A}$ to find the face to which the description refers. If it is a $\mathrm{B}$, look on the page marked group $B$ to find the face to which the description refers. You may use a face as an answer any number of times and need not use each face an equal number of times." The experimenter emphasized these last two points, and encouraged subjects to respond spontaneously.

Groups. Subjects were assigned to one of three groups. Subjects in the neutral context group received booklets containing descriptions that referred only to pictures on the neutral part of the pleasantness dimension (scale values $3.7-6.6$ on a $1.0-9.0$ scale). Subjects in the full-range context group received descriptions that referred to pictures covering the entire pleasantness dimension. Subjects in the extreme group received descriptions that were all based on photographs from the nonneutral parts of the pleasantness scale $(1.0-3.3,7.0-9)$. For each context group the photographs that were described had a mean pleasantness value of 5.0 (the neutral point).

Context and Test Descriptions. In order to directly compare the decoding behavior of subjects in the three context groups, it was necessary to have them decode a common set of test descriptions (descriptions of the same kind as the context descriptions-except test descriptions are the same 
across experimental groups). All three groups were therefore periodically given test descriptions embedded within the context passages. The test descriptions were located on the border between the neutral and extreme areas of the pleasantness scale (3.3 and 6.8).

Each booklet contained sixty-two descriptions arranged in the following sequence: ten context, four test, twenty context, four test, twenty context, four test. Each context set was constructed so that the mean of the pictures to which the descriptions referred was 5.0, the neutral point on the pleasantness scale. Thus, the mean stimulus value was constant for the three context groups, while the variance was manipulated. Each group of four test descriptions may be thought of as a testing time; there are three testing times. The purpose of the "time" variable was to see if the context effect developed gradually during the course of the experiment. The four test descriptions that were included at each testing time were made up as follows: two were slightly on the pleasant side of neutral and two were slightly on the unpleasant side. Of the two on the pleasant side there was one whose appropriate referent (photograph) was located on page $A$ and one from page B; a similar scheme was employed for the two unpleasant test descriptions.

Page. The purpose of the "page" variable was to differentiate between central (perceptual) and peripheral (response bias) interpretations of any context effects that were observed. All of the context descriptions referred to photographs drawn from page $A$ (the subject chooses a match from page A), while half of the test descriptions referred to photographs from page $\mathrm{A}$ and half to photographs from page $\mathrm{B}$. Thus the equal frequency response bias (see above) might affect a subject's reaction to the test descriptions that were derived from page A, but would not affect his reaction to those drawn from page $B$. The biased set of context stimuli did not require that the respondent "overuse" a particular subset of the page $B$ alternatives as matches, while it would require this for a subset of the page A alternatives. As a consequence, the effects that were observed on the page $B$ test trials should be free of the equal frequency response bias, and might more confidently be interpreted as reflecting a central process.

Ordering of Test Descriptions. Test descriptions were placed in four different orders; orders were crossed with the three context groups making twelve types of booklets. Order of presentation of test descriptions was counterbalanced within each testing time.

\section{Data Analysis}

Decoding responses were quantified by assigning the pleasantness value of the chosen photograph (Schlosberg, 1952). In the analysis below, 
Table 1. Pleasantness Values of Photographs Chosen in Response to Test Descriptions (Experiment I)

\begin{tabular}{|c|c|c|c|c|}
\hline \multirow[b]{3}{*}{ Context group } & \multicolumn{4}{|c|}{$\begin{array}{l}\text { Page from which description } \\
\text { drawn }\end{array}$} \\
\hline & \multicolumn{2}{|c|}{ Page A } & \multicolumn{2}{|c|}{ Page B } \\
\hline & \multicolumn{2}{|c|}{$\begin{array}{l}\text { Deviation } \\
\text { of test de- } \\
\text { scription } \\
\text { from } \\
\text { neutral }\end{array}$} & \multicolumn{2}{|c|}{$\begin{array}{l}\text { Deviation } \\
\text { of test de- } \\
\text { scription } \\
\text { from } \\
\text { neutral }\end{array}$} \\
\hline & $-\ldots$ & + & - & + \\
\hline Neutral & 3.84 & 6.81 & 4.16 & 5.51 \\
\hline Full & 4.36 & 6.65 & 4.35 & 6.01 \\
\hline Extreme & 4.47 & 6.31 & 4.14 & 5.72 \\
\hline
\end{tabular}

cell means have been substituted for missing values where subjects did not follow directions (Bennet \& Franklin, 1954, p. 382). Analysis of variance was based on two between-subjects variables: context group and order; and three within subject variables: time, page, and pleasantness. Order was treated as a random effect. ${ }^{4}$ Interactions involving context group and pleasantness value of the test descriptions would be of primary interest in this design, if, as we anticipated, subjects in the neutral context group were induced to choose relatively extreme photographs in response to the test descriptions. More concretely, these subjects should choose particularly pleasant photographs in response to test descriptions that were based on the pleasant emotions, and particularly unpleasant photographs in response to test descriptions that were based on unpleasant emotions.

\section{Results}

Table I presents the pleasantness values of chosen photographs for each context group summed across subjects, time, and orders (higher numbers indicate greater pleasantness). Inspection of these data revealed that decoding behavior followed the predicted pattern for the test descriptions derived from page $A$, but not for the test passages derived from page $B$. On the page A test descriptions, the neutral context group chose the most extreme referents, while the extreme context group chose the most neutral referents.

${ }^{4}$ Strictly speaking, orders were not sampled randomly since they were counterbalanced. However, when order is treated as a fixed effect the results are not substantially different than those presented for any of the three experiments. 
Analysis of variance confirmed the significance of these patterns. First, there was clear evidence of a triple interaction between context groups, response page (A vs. B), and the pleasantness value of the test descriptions $[F(2,6)=11.20, p<.01]$. The group $\mathrm{X}$ pleasantness interaction was not significant. These results indicate that the two response pages yielded different data patterns. To elucidate these patterns, separate analyses were performed for each page. The results from the page A data confirmed the significance of the interaction between context and pleasantness $[F(2,6)=9.43, p<.025]$. By contrast, this same interaction was not significant for the page $\mathrm{B}$ data $(F=.88)$.

In the overall analysis, the group $X$ time $X$ pleasantness interaction proved to be significant $[F(4,12)=4.04, p<.05]$. This effect is difficult to interpret, as it did not appear to reflect the operation of any regular (systematic) process; for example, it did not derive from an increasing separation of the different context groups as time passed (and the contrasting contexts presumably became more firmly established). Our present view is that this unexpected triple interaction derives from an interaction between the various context conditions, and random (uncontrolled) differences between the test passages that were presented at different points in the experimental session.

These results show a clearcut contrast effect due to the variance of the stimulus array. The pattern of the results suggests that this effect probably derived from a response bias, rather than from a more central (perceptual) process. According to this interpretation, responses to the page A descriptions were subject to the equal-frequency bias, since here the subjects had a history of systematically biased responding, based on the context group to which they were assigned. A tendency to select those photographs that had been infrequently "called for" might thus have produced the observed pattern of results. On page B, on the other hand, no history of biased responding had been established, and an equal frequency response bias would have no systematic effect.

\section{EXPERIMENT II}

Experiment II explored the equal frequency response bias in a study that manipulated the "mean" rather than the "variance" of the stimulus array (in accordance with the vast bulk of the context effects literature). Manis (1967) concluded that contextual variations of this type produced effects that were centrally (perceptually) mediated. Experiment II was designed to reassess this hypothesis by replicating Manis' study, with the addition of appropriate controls (see the page variable, above) to assess interpretations involving the equal frequency response bias. 


\section{Method}

\section{Subjects}

Seventy-two volunteers were paid two dollars each.

\section{Apparatus}

Photographs. The same two pages of photographs were used.

\section{Procedure}

Procedure was similar to Experiment I. Subjects were instructed to circle the number of the chosen referent from a row of numbers that appeared below each description. This reduced the missing data rate to zero, since subjects could not choose a referent from the "wrong page," as had happened occasionally during Experiment I.

Groups. Subjects were assigned to one of three groups. Those in the pleasant context group received booklets containing descriptions of pleasant faces, those in the unpleasant context group received booklets containing descriptions of unpleasant faces, and those in the full range context group received booklets containing descriptions of both pleasant and unpleasant faces.

Page. As in Experiment $I$, the page distinction was used to associate different response histories with the sets of referents that appeared on page A and page B. Recall from Experiment I that subjects responded to all of the "context" descriptions by choosing from the photographs that appeared on page A; page B was only used occasionally, to decode half of the test descriptions. Page A might thus be characterized as the "bias" page and page B the "bias-free" page. In Experiment II, for half the subjects page A was the "bias" page and page B was the "bias-free" page, while for the other half of the subjects the reverse was true. Subjects in Experiment II were thus divided into two blocks; by combining responses from these two blocks we constructed separate scores for the "bias" and "bias-free" test trials.

Booklets. As in Experiment I, subjects decoded both context and test descriptions. Test descriptions (embedded within the context series) were common to all three groups and referred to photographs near the neutral point on the pleasantness dimension. There were twelve pages per booklet. Each page had six descriptions: five were context passages and one was a test passage. Test descriptions were placed in each of the serial positions on the various pages. Booklet pages containing test descriptions from the 
"bias" and "bias-free" photograph pages were alternated. In addition, a Latin-square design was employed to counterbalance the order of the pages within booklets. By combining responses over the different orderings within each context condition, we can see time changes averaged over all the "bias" or "bias-free" test descriptions.

\section{Results}

The analysis for Experiment II employed five variables: block (depending on whether A or B was the "bias" page), context group, booklet page ordering, trial (one for each pair of booklet pages), and page (one "bias" and one "bias-free" test description for each trial). This analysis is the same as in Experiment I, except that here we have eliminated the pleasantness factor (whether the test description was on the pleasant or unpleasant side of neutral), as all test descriptions are neutral, and we have added the "blocks" factor.

Examination of the data showed results similar to those of Experiment I: a contrast effect was manifested in response to the test passages associated with the "biased" response alternatives, but there was no reliable effect on the "bias-free" test trials. Table II shows the mean pleasantness values of context group choices; data are averaged across blocks, subjects, orders, and trials, since the $F \mathrm{~S}$ for these factors were all less than 1.00. Those subjects who had decoded unpleasant context descriptions chose the most pleasant faces, while subjects who had decoded pleasant descriptions chose the most unpleasant faces in response to neutral test descriptions.

The effect of the context variable (combining both "bias" and "biasfree" test trials) was not significant $[F(2,10)=2.70, p<.20]$. Note in Table II, however, that most of the difference between the context groups occurs on the "bias" trials; similarly, the group X page interaction was significant $[F(2,10)=7.00, p<.02]$. This interaction suggested the desirability of conducting separate analyses of data from the "bias" and the "biasfree" responses. The analyses confirmed that a contrast effect was operative on the "biased" test trials $[F(2,10)=6.42, p<.025]$, but not on the

Table II. Pleasantress Values of Photographs Chosen in Response to Neutral

Test Descriptions (Experiment II)

\begin{tabular}{lcc}
\hline & \multicolumn{2}{c}{ Test trials } \\
\cline { 2 - 3 } Context Group & Bias & Bias-free \\
\hline Pleasant & 4.58 & 4.98 \\
Full Range & 4.98 & 5.01 \\
Unpleasant & 5.06 & 5.06 \\
\hline
\end{tabular}


"bias-free" trials $(F<1)$. Note, however, that even on the "bias-free" trials the results are ordered in a fashion that is fully consistent with the traditional contrast effect. That is, subjects assigned to the pleasant context selected photographs that were relatively unpleasant, while those assigned to the unpleasant context chose relatively pleasant photographs. As in Experiment I, there is no evidence that the contrast effect was gradually "strengthened" over time; this result is consistent with the data previously reported by Manis (1967).

\section{EXPERIMENT III}

Experiments I and II were consistent in showing the operation of an equal frequency response bias. In both experiments, respondents who had a history of "overchoosing" certain photographs from the "bias" page were more likely to pick "underchosen" photographs from that page when decoding the test descriptions. In dealing with the response alternatives on the "bias-free" page, on the other hand, the subjects had not been induced to "overchoose" a particular subset of the available possibilities. Here one finds scant evidence that the context passages had influenced reactions to the test items, although as noted above, the results from Experiment II were ordered in perfect accordance with the contrast hypothesis, but were far from significant.

While the results of Experiments I and II were consistent in supporting an equal-frequency interpretation for the contrast phenomenon, a reevaluation of these studies suggested that they might provide a less than optimal setting in which to observe the operation of central (perceptual) displacements. Specifically, the multidimensionality of the descriptions and the photographs presented something of a problem. Since these materials varied in many respects, not just in pleasantness, it seemed possible that the decoding choice on a given test trial might be mainly determined by these other dimensions. Thus, even if the apparent pleasantness of a test description had been influenced by the contextual passages that had been previously presented, the respondent's selection of a particular photograph as the referent for that test description might remain unchanged. For example, a photograph might be the only one of the available alternatives that conveyed the proper degree of "alertness," or the only one that could properly be characterized as "smirking." Unique dimensions, apart from the pleasantness of the stimuli, might thus have masked or diluted any central (perceptual) effects. In Experiment III our main goal was to re-establish the contextual contrasts of Experiment II while minimizing the impact of all factors save for the pleasantness dimension, by directing the subject's attention towards pleasantness. 


\section{Method}

One important procedural change was introduced. Instead of asking respondents to choose the appropriate referent for each description (i.e., the photograph on which it was based), the respondents were instructed to match each description to a face that conveyed the same degree of pleasantness. Subjects were thus instructed to (a) read each passage, (b) decide on the pleasantness of the emotion that was being described, and then (c) indicate which of the various faces on a given page seemed an appropriate "match" (ignoring everything else about the passages and the photographs, apart from their pleasantness).

In order to reduce the unique pull of the correct photograph, for each description, the set of photographs ("page") from which the respondent was to make this choice was switched. That is, for descriptions that were based on faces from page $A$, we asked the respondents to choose a face from page $B$ that seemed equal to the original referent in terms of pleasantness, and vice versa.

\section{Results and Discussion}

Analysis of these data was the same as for Experiment II. The results, however, were somewhat different. Most importantly, the linear trend associated with the context manipulation showed clearcut evidence of a contrast effect $[F(1,10)=6.32, p<.05]$, that is, the respondents who had been assigned to the pleasant context group chose less pleasant photographs in matching test passages than the respondents who were in the unpleasant context group, while the full-range group occupied an intermediate position in this regard. In addition, the interaction of context $X$ page which played such an important role in Experiment I and II was small $(F<1)$. The trials variable did not interact with the context manipulation.

The absence of a significant context $X$ page interaction suggests that the contrast effect was reliable whether the respondent was evaluating a test passage that required a choice from the "bias" page (where response biases might be operative) or a passage that required him to choose from the "bias-free" page (where response biases were not operative). Inspection of Table III indicates, nonetheless, that the contrast effect was considerably stronger in the trials that involved the "bias" page; indeed, when considered separately, the results that were obtained on the "bias-free" page were not significant, while the trials that involved the "bias" page proved to be reliable. Following the conservative logic that is normally applied in cases like this, however, we would conclude that the type of test trial did not reliably affect the results of this experiment (since there was no evidence of a context $X$ page interaction). Hence, a central (perceptual) interpretation 
Table III. Pleasantness Values of Photographs Matched to Neutral Test Descriptions (Experiment IH)

\begin{tabular}{lccc}
\hline & \multicolumn{3}{c}{ Test trials } \\
\cline { 2 - 4 } Context Group & Bias & Bias-free & Combined \\
\hline Pleasant & 5.00 & 4.99 & 5.00 \\
Full Range & 5.18 & 5.21 & 5.20 \\
Unpleasant & 5.42 & 5.20 & 5.31 \\
\hline
\end{tabular}

would seem to fit these data most parsimoniously, since the observed effects were apparently quite general (see footnote 3 ).

The results from Experiments II and III present an interesting contrast. When these experiments were analyzed separately they yielded rather different results: Experiment II suggested that the equal frequency response bias was a significant determinant of performance, while Experiment III indicated that it was not (the interaction between context group and page was far from significant in Experiment III). An overall analysis combining the data from the two experiments indicated, however, that any differences between the two data sets could easily be attributed to random error.

The results from this overall analysis (combining the data of Experiments II and III) suggest the following conclusions:

1. While the context manipulations had a clear effect on performance $(p<.001)$, the observed contrast pattern was primarily observed on trials where the equal frequency bias was left uncontrolled (the interaction between context group and page had a $p$ value less than .10).

2 . Nonetheless, a separate analysis of the data from the bias-free trials of Experiments II and III combined provided some support for the proposition that the observed effects were not solely attributable to the equal frequency bias. In this subset of the combined data, the results from the three context groups fell into a pattern that was perfectly consistent with the classic contrast effect (subjects assigned to the unpleasant context group selected the most pleasant referents on the test trials, while those in the pleasant context condition chose the least pleasant referents). Moreover, a trend analysis of these data indicated that the linear component of the context variable was marginally significant $[F(1,48)=2.53, p=.06$, onetailed test] suggesting that the contrast effect may have been partly mediated by central (perceptual) processes.

\section{CONCLUSION}

The results of these three experiments suggest that context effects often derive from an equal frequency response bias. Thus, in judging a series of discrete stimuli (descriptive passages in the present studies), 
changes in the stimulus array may produce reliable effects that result from the subject's implicit attempt to use each of the available response alternatives with roughly equal frequency. It is interesting to note that this response bias appeared with relative clarity despite an explicit instruction that the subjects should not be concerned about the frequency with which any particular response seemed to be "called for."

Neither our studies, nor that of Manis (1967), showed any sign of an increment in context-induced effects as the experimental session progressed. That the context effect should appear full blown, with virtually no warm-up time is somewhat surprising. Further research will be needed to clarify this puzzling phenomenon.

The conflict between response-based and central (perceptual) explanations has arisen in many domains. The issue is still important, for example, in perceptual defense (Erdelyi, 1974). The breadth and long history of this conflict contribute to its importance. The present experiments suggest that response processes contribute importantly to context effects. Central (perceptual) processes may also play some role in situations where the central tendency of a stimulus set has been drastically altered, as in Experiments II and III, although the evidence here is less clearcut. In our research, a response bias was examined by the introduction of two separate response languages (the "pages"); when response effects are assessed in this way, central processes may be more plausibly demonstrated. Evidence for the role of central (perceptual) processes has also recently been reported by Simpson and Ostrom (1976) in an experiment that focused on contextual ef fects in impression formation, and in Krupat (1974), where skin resistance (BRL) was used to assess perceived threat.

Reviewing the long history of this issue, it may be fruitful to suggest that the classic dispute between central (perceptual) and peripheral (response-bias) interpretations of contextual phenomena should now be recast in a form that encourages research concerning the situation and subject variables that are conducive to the effective demonstration of these separate processes.

\section{ACKNOWLEDGMENT}

The authors wish to thank Dr. Daniel Weintraub and Dr. Donald R. Brown for comments on an earlier version of this paper.

\section{REFERENCES}

Bennett, C., \& Franklin, N. Statistical analysis in chemistry and the chemical industry. New York: Wiley, 1954. 
Campbell, D. T., Hunt, W. A., \& Lewis, N. A. The relative susceptibility of two rating scales to disturbances resulting from shifts in stimulus context. Journal of Applied Psychology, 1958, 42, 212-217.

Erdelyi, M. H. A new look at the new look: Perceptual defense and vigilance. Psychological Review, 1974, 81, 1-25.

Frois-Wittman, J. The judgment of facial expression. Journal of Experimental Psychology, $1930,13,113-151$.

Helson, H. Adaptation-level theory. New York: Harper \& Row, 1964.

Krantz, D. L., \& Campbell, D. T. Separating perceptual and linguistic effects of context shifts upon absolute judgments. Journal of Experimental Psychology, 1961, 62, 35-42.

Krupat, E. Context as a determinant of perceived threat: The role of prior experience. Journal of Personality and Social Psychology, 1974, 29, 731-736.

Manis, M. Context effects in communication. Journal of Personality and Social Psychology, $1967,5,326-334$.

Manis, M. Context effects in communication: Determinants of verbal output and referential decoding. In M. H. Appley (Ed.), Adaptation level theory. New York: Academic Press, 1971.

Manis, M., \& Armstrong, G. A. Contrast effects in verbal output. Journal of Experimental Social Psychology, 1971, 7, 381-388.

Parcucci, A. The relativism of absolute judgments. Scientific American, 1968, 219(6), 84-90.

Parducci, A. Contextual effects: A range-frequency analysis. In E. C. Carterette \& M. P. Friedman (Eds.), Handbook of perception, Vol. II, New York: Academic Press, 1974.

Simpson, P., \& Ostrom, T. Contrast effects in impression formation. Journal of Personality and Social Psychology, 1976, 34, 625-629.

Schlosberg, H. The description of facial expression in terms of two dimensions. Journal of Experimental Psychology, 1952, 44, 229-237.

Upshaw, H. S. The personal reference scale: An approach to social judgment. In L. Berkowitz (Ed.), Advances in experimental social psychology, Vol. 4, New York: Academic Press, 1967. 